\title{
Complexities and challenges of transition to adult services in adolescents with vertically transmitted HIV infection
}

\section{Ruth Harris}

Medical Student, MBBS Year 5, King's College London, London, UK

\section{Correspondence to} Ms Ruth Harris, King's College London, London SE1 1UL, UK; ruth.e.harris@kcl.ac.uk

Received 9 June 2014 Revised 29 October 2014 Accepted 3 November 2014
CrossMark

To cite: Harris R. J Fam Plann Reprod Health Care 2015;41:64-67.

\section{BACKGROUND}

Increasing numbers of HIV-positive young people are transitioning from paediatric to adult services. According to the latest Collaborative HIV Paediatric Study (CHIPS) report ${ }^{1}$ there are 1873 children with vertically acquired HIV (HIV acquired by mother-to-child transmission) in UK and Ireland, with 595 young people transitioning to adult services. There have been 50-100 young people transferring each year between 2007 and 2013, with a median age of 17.5 (interquartile range 16.6-18.3) years. ${ }^{1}$ Those aged $>15$ years have increased from $<1 \%$ in 1996 to $40 \%$ in 2012. ${ }^{1}$ The development of this vulnerable new patient group means that health care services providers and planners need to take into account the changing needs of these young people living into adulthood with HIV.

High uptake of antenatal testing, access to highly active antiretroviral treatment (HAART) and effective intervention in preventing mother-to-child transmission in well-resourced countries such as the UK means that perinatally acquired HIV rates are very low. Young people growing up with vertically transmitted HIV in the UK are predominantly of sub-Saharan African origin and born outside the UK. ${ }^{1}$ There is a trend towards increasing age of newly diagnosed HIV-positive children living in the UK but born abroad, because it has become apparent that 1 in 10 of infected children are predicted to survive without treatment up to 10 years of age. ${ }^{2}$ These are the evolving reasons for the growing number of children and young people with vertically transmitted HIV in the UK.

The development of effective treatment means that children with vertically acquired HIV are now expected to survive beyond childhood. According to a report by the Antiretroviral Therapy Cohort Collaboration $^{3}$ involving meta-analysis of 14 cohorts of HIV-positive adolescents, a 20-year-old starting antiretroviral treatment could expect to live an additional 43 years. The success of HAART and the recognition of the epidemic have meant that more young people are living and growing up with HIV in the UK and this picture is likely to become increasingly common. This new expectation means that such children face the usual challenges of adolescence in addition to the complex medical needs and psychosocial issues associated with their HIV status. This essay will focus on summarising some of these issues with particular focus on sexual and reproductive health (SRH) in HIV-positive young people and the challenges faced in transition to adult health care services.

\section{COMPLEXITIES SURROUNDING HIV INFECTION IN ADOLESCENTS}

HIV in the adolescent is a complex area. Adolescence in itself is a period of transition, both physically and mentally. It is characterised by development of an identity, greater social autonomy, biological and sexual maturity, and increased competency. Issues that face all teenagers and young people are exaggerated when the individual has a chronic disease as allencompassing and with such wide social and sexual implications as HIV.

According to Lyall: ${ }^{4}$ "Transition of care from a paediatric to adult setting is not specific to HIV, but occurs in a number of paediatric specialities ... However, there are important differences for young people with HIV which may make this process more difficult”. These differences include issues about disclosure, sexual health and 
relationships, stigma, and psychological and physical health.

The stigma attached to the diagnosis for the parents, family and wider community can restrict the individual from knowing their HIV status until they are older. Disclosure is the term used when the HIV-positive person is told their HIV status. In the adolescent it is a crucial prerequisite to becoming independent in transition. It is the foundation to knowing about HIV and being able to live with and adhere to medication as an adult. Confidentiality is paramount when treating and caring for those with HIV regardless of their age. There is an important trust relationship between the individual and the health care team about sharing information and listening to what information the patient would like to be shared and with whom.

Adolescents born with HIV have to deal with a sexually transmitted infection (STI) before having sex themselves. They have an added pressure of negotiating sexual relationships with others in a mature and safe manner. "For young people with HIV, as interest in sexual relationships develops, the risk of onward transmission of the virus also requires that they exhibit a higher degree of self-confidence, responsibility and maturity than their peers in negotiating relationships." ${ }^{5}$ During transition of a young person with HIV, it is key that they are informed about their sexual health, given accurate information, and a safe space to discuss and ask questions before they embark on any sexual experiences. Information that should be discussed includes legal issues around non-disclosure and transmission, use of contraception options that are available to them on their individual HAART, ${ }^{6}$ and an awareness of post-exposure prophylaxis after sexual exposure to HIV (PEPSE). ${ }^{7}$

The sexual activity of young people with vertically transmitted HIV is a key concern for SRH services. According to a recent HIV Young Persons Network (HYPNet) survey, 57\% of the adolescent cohort were sexually active, of whom one-quarter reported suboptimal condom use and one-fifth had had a past STI. ${ }^{9}$ Negotiating safer sex for all young people is important and the lack of condom use is reflected in a study of 172 young women growing up with HIV of whom 36 reported pregnancy, with 27 (75\%) of these being unplanned. ${ }^{10}$ In addition to this, a major challenge for young people with HIV is the limited contraceptive options that are available to them, considering the interactions and altered efficacy with current antiretroviral treatments (see UK Medical Eligibility Criteria for Contraceptive Use ${ }^{6}$ for guidance). Little is known about the reproduction and fertility of this new vulnerable group growing up with HIV. Data and research are crucial to improving targeted sexual and reproductive health services as well as raising awareness of the unique needs and protection of young people in the UK with vertically transmitted HIV.
A diagnosis of HIV is still stigmatising and this makes living with this disease for young people especially complex. They may feel unable to disclose to their peers and may find conversations at school or elsewhere hard to tackle. There is the added burden of family relationships, which can be stressful for most teenagers. The coming to terms with having a vertically transmitted infection can affect the relationship with their mother and other family members who may or may not also have HIV. Transition in HIV has a wider role in building up the individual's self-esteem and independence in the context of the family and peer support available. This is not always possible and presents a challenge to the young person with HIV and the multidisciplinary transition service that is caring for them during the transition process.

HIV is an illness that can be fatal, and in order to avoid health complications and hospitalisation young people with HIV should have a clear knowledge about their illness. In addition, understanding HIV for the individual helps them to adhere to life-saving medication and to be able to inform health professionals of any health problems early. Adherence is low in some adolescents compared to adults and children with HIV and the reason for this is multifactorial. ${ }^{11}$ Remembering to take medication when away or out for long periods, especially for an illness for which they may not be showing physical signs or that their peers do not know about, and could possibly shun them if they were to know, can be near impossible for teenagers. Research has shown that the secrecy and stigma that surrounds their diagnosis is considered a barrier to adherence to treatment; ${ }^{12}$ however, it is this medication that is allowing them to live into adulthood. HIV is a hugely complex disease for all those diagnosed with it, but it is especially difficult for adolescents when faced with it at an uncertain time of change. Unlike other chronic illnesses - such as diabetes and cystic fibrosis - HIV presents the individual, family and the health care team with numerous unique challenging and complex issues that are interlinked and must be approached in the transition process.

Adherence to life-saving medication is vital in young people with HIV. An undetectable viral load will dramatically improve health outcomes and life expectancy, and in addition it can reduce the risk of onward transmission of HIV both horizontally and vertically. $^{7}$ It is particularly important to discuss adherence with young people because, as has already been discussed, this population group has the added challenges of disclosure and negotiating condom use. Recent mortality statistics in young people with HIV have shown that 8 out of 11 deaths in this population are due to poor adherence and advanced HIV. ${ }^{13}$ These are shocking data and make the role of effective and flexible transition services ever more important. 
TRANSITION

Transition is the "purposeful, planned process that addresses the medical, psychosocial and educational/ vocational needs of adolescents and young adults with chronic physical and medical conditions as they move from child-centred to adult-orientated health care systems". ${ }^{14}$ When young people are diagnosed with a chronic illness it inevitably means they have ongoing relationships with health care providers as they grow up. These services have to be remodelled but continued successfully and seamlessly from paediatric to adult care. The Department of Health wants to see "young people taking responsibility for their own health and making informed choices and decisions regarding their emotional and social development, and health and well-being both now and in the future". ${ }^{15}$ Transition should be "planned and purposeful"; it is a process, not a time frame. It should allow the individual not simply to move into adult care, but be equipped and skilled to take responsibility and care of their own health.

There are many chronic illnesses diagnosed in childhood such as type 1 diabetes mellitus, renal disease and cystic fibrosis that, with the development of successful treatments, have meant a better prognosis and more people living to adulthood. Like HIV, they have had an emerging role in transition. There are numerous publications about both health care teams' and patients' experiences of transition in other chronic illnesses. Those involved in the HIV transition process can learn from other chronic illnesses' longer history of managing adolescent patients. Bringing these experiences together, McDonagh attempted to develop one model of transition care for young people that could be applied universally to a range of chronic illnesses. ${ }^{16}$ Common themes that were identified included:

- An active future-focused process

- Young person centred

- Inclusive of parents/caregivers

- Multidisciplinary and interagency.

Overall, McDonagh was not able to emphasise one overarching model of transition being better than another, but rather transition being a preparation process that suits the needs of the individual that is flexible and that starts early. If transition is not done well - for example, being done abruptly or without adequate preparation - patients may withdraw from medical care altogether, and this is both detrimental to the individual's health and an expense to the health care services overall.

The Children's HIV Association of the UK and Ireland (CHIVA) has integrated transition planning and process into their guidelines. CHIVA is a body of professionals committed to improving standards of care for children with or affected by HIV. Standard 9 in 'The CHIVA Standards of Care for Infants, Children and Young People Living with HIV ${ }^{17}$ states that the transition process should happen over time and be appropriate to the individual's age, development and circumstances. These standards are guidance to transition and can aid the process; however, the role of transition in HIV is a relatively novel one and as yet in the UK it is not a well understood or researched concept.

There has been recognition of the lack of research in HIV transition services. Sexual health services are starting to assess the established clinics that care for adolescents with vertically transmitted HIV. The Caldecot Centre, the sexual health department at King's College Hospital, set up a new young persons' HIV clinic in 2011. A recent audit and patient satisfaction questionnaire illustrated successes in the eyes of the young people, such as readily available sexual health advice, accessible contraception and being seen in a confidential service. ${ }^{18}$ However, high rates of non-attendance for appointments were also highlighted, in keeping with experiences of other adolescent services. Analysing what young people want from their transition services has shown that it is paramount that the young person feels that they have the support and care in an individual and personal manner. ${ }^{19}$ Collaborative working as a team with the young person at the centre of this is vital to maintain a safe and open environment, allowing the young person to share their concerns. More data are needed in order to effectively evaluate the services being provided and to ensure that the complex needs of caring for those young people with HIV are being met.

\section{CONCLUSIONS}

There are numerous challenges and complexities to transition in adolescents with vertically transmitted HIV infection. HIV is a unique chronic illness in young people in the UK. With current antenatal prevention and treatment it is rare to have HIV-positive children born in the UK. The development of effective HAART has ensured that there are more adolescents living on to adulthood with HIV. Health care services that work with this cohort are relatively new to the transition process. There will be an increased need for these services to be assessed and improved as well as to learn from other more established chronic diseases that have had longer experience with the transition process.

Transition services that are available to young people with HIV should be fully aware of the complexities that caring for this cohort entails. Transition should be a planned and purposeful process that takes into account the individual and his or her wider needs. A multidisciplinary and interagency approach is recommended, and continuous reflection and change should occur.

Editor's note This is a revised and extended version of the joint winning entry for the 2014 FSRH Margaret Jackson Prize Essay for medical students. 
Acknowledgements The author would like to thank the Journal's peer reviewer and Dr Elizabeth Hamlyn for their helpful comments that have strengthened this essay, and Dr Elizabeth Hamlyn and Dr Colin Ball for the opportunity to carry out a placement in adolescent HIV at King's College Hospital, London, UK.

\section{Competing interests None.}

Provenance and peer review Not commissioned; externally peer reviewed.

\section{REFERENCES}

1 Collaborative HIV Paediatric Study (CHIPS) Annual Report 2013/2014, July 2014. http://www.chipscohort.ac.uk [accessed 1 November 2014].

2 Judd A, Ferrand R, Jungmann E, et al. Vertically acquired HIV diagnosed in adolescence and early adulthood in the UK and Ireland: findings from national surveillance. HIV Med 2009;10:253-256.

3 Antiretroviral Therapy Cohort Collaboration. Life expectancy of individuals on combination antiretroviral therapy in high-income countries: a collaboration analysis of 14 cohort studies. Lancet 2008;372:293-299.

4 Lyall H. Growing Up, Gaining Independence Principles for Transition of HIV Care. 2007. http://www.chiva.org.uk/files/ guidelines/growing-up09.pdf [accessed 1 November 2014].

5 Howell S, Hamblin E. Children and Young People HIV Network, National Children's Bureau. Young People Living with HIV and the Transition from Children's to Adult Services: Literature Review. 2011. http://www.ncb.org.uk/media/233249/ yp_living_with_hiv.pdf [accessed 1 November 2014].

6 Faculty of Reproductive \& Sexual Health Care. UK Medical Eligibility Criteria for Contraceptive Use (UKMEC 2009). 2009. www.fsrh.org/pdfs/ukmecsummarysheets2009.pdf [accessed 1 November 2014].

7 Williams I, Churchill D, Anderson J, et al. British HIV Association (BHIVA) Guidelines for the treatment of HIV-1 positive adults with antiretroviral therapy 2013. HIV Med 2014;15(Suppl. 1):1-85.

8 Benn P, Fisher M, Kulasegaram R, on behalf of BASHH PEPSE Guidelines Writing Group Clinical Effectiveness Group. UK Guideline for the use of post-exposure prophylaxis for HIV following sexual exposure. Int J STD AIDS 2011;22:695-708.

9 Barbour A, Hamlyn E, Foster C, et al. Sexual and reproductive health (SRH) amongst HIV positive adolescents: an HIV Young Persons Network (HYPNET) survey. Poster Presentation P420. British HIV Association Conference 2014. HIV Med 2014;15 (Suppl. 3):17-159.
10 Kenny J, Williams B, Prime K, et al. Pregnancy outcomes in adolescents in the UK and Ireland growing up with HIV. HIV Med 2012;13:304-308.

11 Foster C, McDonald S, Griffiths J, et al. HYPNET Adherence Guidelines for Adolescents and Young Adults Living with HIV. 2014. http://www.hypnet.org.uk/pages/guidelines.php [accessed 1 November 2014].

12 McDonald S, Griffiths J, Tanna N, et al. Young people and self-reported adherence to antiretroviral therapy: a HYPNet survey. Poster Presentation P198. British HIV Association Conference 2011. HIV Med 2011;12(Suppl. 1): $14-86$.

13 Fish R, Jungmann E, Judd A, et al. Mortality amongst HIV-infected young people following transition to adult care: an HIV Young Persons Network (HYPNet) audit. Poster Presentation 06, British HIV Association Conference 2012. HIV Med 2012;13(Suppl. 1):1-11.

14 Department of Health. National Service Framework for Children, Young People and Maternity Services. Core Standards. 2004. https://www.gov.uk/government/uploads/system/uploads/ attachment_data/file/199952/National_Service_Framework_ for_Children_Young_People_and_Maternity_Services_-_Core Standards.pdf [accessed 1 November 2014].

15 Department of Health. National Service Framework for Children, Young People and Maternity Services. Transition: Getting it Right for Young People. 2006. http://webarchive. nationalarchives.gov.uk/20130107105354/http://www.dh.gov. uk/prod_consum_dh/groups/dh_digitalassets/@dh/@en/ documents/digitalasset/dh_4132149.pdf [accessed 1 November 2014].

16 McDonagh J. Lost in transition? Between paediatric and adult services: it's time to improve the transition of adolescents from paediatric to adult services. BMJ 2006;332:435-436.

17 Lyall H, Riordan A, Williams A. The CHIVA Standard of Care for Infants, Children and Young People with HIV (including infants born to mothers with HIV). Standard 9: Transition to Adolescent \& Adult HIV Specialist Services. 2010. http://www. chiva.org.uk/professionals/health/guidelines/guidelines/ standards/2013/transition.html [accessed 1 November 2014].

18 Harris R, Campbell L, Callaghan A, et al. Growing up with HIV: what do young people want from their transition clinic? Poster Presentation P23. British HIV Association Conference 2014. HIV Med 15(Suppl. 3):17-159.

19 Bundock H, Fidler S, Clarke S, et al. Cross the divide: transition care services for young people with HIV-their views. AIDS Patient Care STDS 2011;25:465-473. 